\title{
Comunicação
}

[Communication]

\section{Frequência de parasitos intestinais em cães domiciliados da cidade de Maringá, PR}

[Frequency of intestinal parasites in domiciled dogs from Maringá city, Brazil]

\author{
L. Prates $^{1}$, L.S. Pacheco ${ }^{1}$, J.B. Kuhl ${ }^{2}$, M.L.G.G. Dias $^{3}$, S.M. Araújo ${ }^{3}$, A.R.T. Pupulin ${ }^{3 *}$ \\ ${ }^{1}$ Aluna de pós-graduação - UEM - Maringá, PR \\ ${ }^{2}$ Laboratório de Parasitologia Básica - UEM - Maringá, PR \\ ${ }^{3}$ Universidade Estadual de Maringá - UEM - Maringá, PR
}

Animais de estimação, particularmente cães e gatos, são importantes companheiros em muitas casas, contudo oferecem risco à saúde dos seus donos pela possibilidade de transmitirem doenças que incluem as parasitárias (Robertson et al., 2000). A relação íntima entre animais domésticos e proprietários resulta na participação em comum de mais de 60 espécies de parasitos como: Giardia, Cryptosporidium, Toxoplasma, Diphyllobothrium, Echinococcus spp., Ancylostoma sp. e Toxocara sp. (MacPherson, 2005). Dado o estreito convívio dos cães com o homem, torna-se fundamental o controle adequado da endoparasitose canina, com o objetivo de diminuir a contaminação do meio ambiente pelas formas infectantes e, consequentemente, minimizar os riscos de infecção humana e canina (Robertson et al., 2000). O objetivo deste estudo foi determinar a frequência de parasitos intestinais em cães de residências na cidade de Maringá, PR.

Foram obtidas amostras fecais de cães de diferentes raças de ambos os sexos e de idades variadas, de famílias residentes na periferia da cidade de Maringá-PR. O tamanho da amostra foi calculado pelo programa EpiInfo 2002 versão 6.0. O bairro estudado foi escolhido mediante sorteio. As fezes foram processadas pelas técnicas de centrífugo-flutuação em sulfato de zinco (Faust et al., 1939) e sedimentação espontânea (Hoffmann et al., 1934). A pesquisa foi aprovada pelo Comitê de Ética em Pesquisa da Universidade Estadual de Maringá.
Durante o período novembro de 2006 a novembro de 2007, foram examinadas fezes de 81 cães pertencentes a 73 famílias. Das amostras fecais examinadas, 44,4\% (36/81) foram positivas para ovos e cistos de enteroparasitos. Os mais frequentes foram Ancylostoma spp., com 18,5\% (15/81), e Giardia lamblia, com 11,1\% (9/81). Os demais parasitos tiveram frequências abaixo de $10 \%$.

O resultado encontrado neste estudo está dentro do registro mundial de prevalências de helmintos intestinais em caninos entre $4 \%$ e $78,0 \%$, determinadas por meio de análises em material fecal e em inspeção post mortem (Giraldo et al., 2005).

$\mathrm{O}$ parasito mais frequente encontrado, Ancylostoma spp., 18,5\%, foi também registrado por Costa et al. (1990), em Vitória, ES, 88,5\%, por Oliveira-Sequeira et al. (2002), em Botucatu, SP, 31,9\%, por Vasconcelos et al. (2006), no Rio de Janeiro, RJ, 34,8\%, por Gennari et al. (1999), em São Paulo, SP, 20,40\%, e por Araújo (2006), em Viçosa, MG, 18,53\%. Segundo Labruna et al. (2006), mesmo diante das diferenças metodológicas entre os inquéritos parasitológicos realizados no Brasil, Ancylostoma spp. foi sempre o gênero de helminto mais frequentemente relatado em cães. Ancylostoma spp. tem seus ovos comumente disseminados pelo solo, o que explicaria a prevalência encontrada. 
A frequência para G. lamblia, 11,1\%, é semelhante à registrada por Funada et al. (2007), $8,5 \%$, e por Labruna et al. (2006), 8,4\%. Esta frequência é preocupante, pois, embora esse protozoário seja de pouca significância clínica para os cães e evidências epidemiológicas indiquem que eles desenvolvem resistência ao parasito em consequência da exposição prévia e/ou da maturação do sistema imunológico (Oliveira-Sequeira et al., 2002), pesquisas têm mostrado que alguns genótipos do parasito podem ser compartilhados entre humanos e cães, particularmente em áreas urbanas. Deve-se ressaltar que os cistos de $G$. lamblia são resistentes ao tratamento da água e podem permanecer viáveis na superfície por aproximadamente dois meses (MacPherson, 2005).

Ovos de Trichuris vulpis foram encontrados em $6,2 \%$ das amostras examinadas. Frequências maiores que a observada neste estudo foram registradas por Costa et al. (1990), 24,5\%, Scaini et al. (2003), em Balneário Cassino, RS, 32,5\%, Capuano e Rocha, (2006), em Ribeirão Preto, SP, $15,7 \%$, e Labruna et al. (2006), em Monte Negro, RO, 9,5\%.

Isospora spp., sinônimo de Cystoisospora spp., segundo Oliveira-Sequeira et al. (2002), foi observado com frequência de $3,7 \%$ e também em outros estudos semelhantes, com frequências de 2,6\% (Gennari et al., 1999), 5,9\% (Vasconcelos et al., 2006) e 4,4\% (Funada et al., 2007).

O parasito Toxocara canis é considerado um dos mais comuns mundialmente, e sua prevalência pode chegar até $81 \%$ da população de cães. A frequência encontrada neste estudo foi de apenas $2,5 \%$. Este resultado é semelhante aos encontrados por Costa et al. (1990), 3,2\%, Giraldo et al. (2005), no departamento de Quindio, na Armênia, Colômbia, 2,5\%, e Funada et al. (2007), em São Paulo, SP, 2,6\%. T. canis infecta principalmente cães jovens por transmissão transplacentária e transmamária, isto é, mesmo antes de nascerem e desde o início da amamentação (Trillo-Altamirano et al., 2003; Giraldo et al., 2005). Cães adultos continuam suscetíveis à infecção por T. canis, mesmo quando são repetidamente expostos ao parasito e quando produzem anticorpos específicos. Desse modo, podem contribuir significativamente para a contaminação do ambiente com ovos (OliveiraSequeira et al., 2002).

Os parasitos com menor frequência foram Capillaria spp. e Taenia spp., ambos com 1,2\% cada. Em estudos semelhantes, Costa et al. (1990) encontraram prevalência de $1,6 \%$, e Trillo-Altamirano et al. (2003), na cidade de Ica, no Peru, $4,3 \%$.

Em 33,3\% (27/81) das amostras, foi encontrada apenas uma espécie de parasito, enquanto em $11,0 \%$ (9/81), dois ou mais parasitos. Os negativos representaram 55,6\% (45/81). A associação mais frequente foi Ancylostoma spp. e T. vulpis, $6,2 \%$, o que se justifica pelo fato de esses parasitos estarem entre os mais frequentes na população estudada. Na pesquisa de Leite et al. (2004), em Curitiba, PR, foram observadas associações entre Ancylostoma spp. e Toxocara spp., 3,8\%, seguida de Ancylostoma spp. e $T$. vulpis, 2,6\%. Também no trabalho de Araújo (2006), a principal associação verificada foi de Ancylostoma spp. e Toxocara spp., 4,8\%, enquanto Scaini et al. (2003) encontraram Ancylostoma spp. e Trichuris spp., 16,9\%.

Também foram encontradas associações de $G$. lamblia e T. canis; Ancylostoma spp. e Taenia spp.; G. lambia, T. canis e Isospora spp.; e Capillaria spp., T. canis e Ancylostoma spp., todas com frequência de $1,2 \%$.

As espécies e os gêneros de parasitos encontrados assemelham-se aos dados da literatura, embora as informações sobre infecções humanas por esses parasitos sejam escassas no Brasil (Oliveira-Sequeira et al., 2002).

Os resultados mostram que os cães domiciliados em bairro da periferia da cidade de Maringá encontram-se infectados por parasitos com elevado potencial zoonótico, com risco para a saúde dos animais e das pessoas que com eles convivem.

Palavras-chave: cão, parasitos intestinais, Maringá 


\begin{abstract}
The frequency of eggs and cysts of intestinal parasites in domiciled dogs that lived in the city of Maringá, Brazil, was studied. From November 2006 to November 2007, stools of 81 dogs were examined, of which $44.4 \%$ were positive for some kind of enteric parasite. The most frequent was Ancylostoma spp. with $18.5 \%$ (15/81) followed by Giardia lamblia with 11.1\% (9/81). There was association of parasites in $11.0 \%$ of the samples, being the most frequent Ancylostoma spp. and Trichuris vulpis (6.2\%) (5/81). There were sources of contamination where the animals lived which were responsible for maintaining the parasite in the environment.
\end{abstract}

Keywords: dog, intestinal parasites, Maringá

\section{REFERÊNCIAS BIBLIOGRÁFICAS}

ARAUJO, J.V. Helmintoses intestinais em cães da microrregião de Viçosa, Minas Gerais. Rev. Ceres, v.53, p.362-364, 2006.

CAPUANO, D.M.; ROCHA, G.M. Ocorrência de parasitas com potencial zoonótico em fezes de cães coletadas em áreas públicas do município de Ribeirão Preto, SP, Brasil. Rev. Bras. Epidemiol., v.9, p.81-86, 2006.

COSTA, J.O.; GUIMARÃES, M.P.; LIMA, W.S. et al. Frequência de endo e ecto parasitos de cães capturados nas ruas de Vitória - ES Brasil. Arq. Bras. Med. Vet. Zootec., v.42, p.451452, 1990.

FAUST, E.C.; SAWIT, W.; TOBEI, J. Comparative efficiency of various techniques for the diagnosis of protozoa and helmints in feces. Int. J. Parasitol., v.25, p.241-62, 1939.

FUNADA, M.R.; PENA, H.F.J.; SOARES, R.M. et al. Frequência de parasitos gastrintestinais em cães e gatos atendidos em hospital-escola veterinário da cidade de São Paulo. Arq. Bras. Med. Vet. Zootec., v.59, p.1338-1340, 2007.

GENNARI, S.M.; KASAI, N.; PENA, H.F.J. et al. Ocorrência de protozoários e helmintos em amostras de fezes de cães e gatos da cidade de São Paulo. Braz. J. Vet. Res. Anim. Sci., v.36, p.87-91, 1999.

GIRALDO, M.I.; GARCIA, N.L.; CASTAÑO, J.C. Prevalencia de helmintos intestinales en caninos del departamento del Quindío. Biomedica, v.25, p.346-352, 2005.

HOFFMANN, W.A.; PONS, J.A.; JANER, J.L. The sedimentation - concentration method in Schistosomiasis mansoni. Am. J. Public Health, v.9, p.281-298, 1934.
LABRUNA, M.B.; PENA, H.F.J.; SOUZA, S.L.P. et al. Prevalência de endoparasitas em cães da área urbana do município de Monte Negro, Rondônia. Arq. Inst. Biol. São Paulo, v.73, p.183-193, 2006.

LEITE, L.C.; MARINONI, L.P.; CÍRIO, S.M. et al. Endoparasitas em cães (Canis familiaris) na cidade de Curitiba - Paraná - Brasil. Arch. Vet. Sci., v.9, p.95-99, 2004.

MacPHERSON, C.N.L. Human behaviour and the epidemiology of parasitic zoonoses. Int. J. Parasitol., v.35, p.1319-1331, 2005.

OLIVEIRA-SEQUEIRA, T.C.G.; AMARANTE, A.F.T.; FERRARI, T.B. et al. Prevalence of intestinal parasites in dogs from São Paulo State, Brazil. Vet. Parasitol., v.103, p.19-27, 2002.

ROBERTSON, I.D.; IRWIN, P.J.; LYMBERY, A.J. et al. The role of companion animals in the emergence of parasitic zoonoses. Int. J. Parasitol., v.30, p.1369-1377, 2000.

SCAINI, C.J.; TOLEDO, R.N.; LOVATEL, R. et al. Contaminação ambiental por ovos e larvas de helmintos em fezes de cães na área central do Balneário Cassino, Rio Grande do Sul. Rev. Soc. Bras. Med. Trop., v.36, p.617-619, 2003.

TRILLO-ALTAMIRANO, M.P.; CARRASCO, A.J.; CABRERA, R. Prevalencia de helmintos enteroparasitos zoonoticos y factores asociados en Canis familiaris em uma zona urbana de la ciudad de Ica, Perú. Parasitol. Latinoam., v.58, p.136-141, 2003.

VASCONCELLOS, M.C.; BARROS, J.L.; OLIVEIRA, C.S. Parasitas gastrointestinais em cães institucionalizados no Rio de Janeiro, RJ. Rev. Saúde Pública, v.40, p.321-323, 2006. 